\title{
Experimental and Guided Theoretical Investigation of Complex Reaction Mechanisms in a Prins Reaction of Glyoxylic Acid and Isobutene
}

\author{
Gaetano Angelici, * Stefan Nicolet, Narasimha R. Uda. Marc Creus*
}

5 Affiliation and address Department of Chemistry, University of Basel, Spitalstrasse 51

CH-4056 Basel, Switzerland

\section{ABSTRACT}

A laboratory experiment was designed for undergraduate students, in which the outcome of an easy single-step synthesis with well defined conditions was not elucidated until the end of the exercise. The laboratory component, divided in two sessions and completed in 6-7 h, was carried out using a Guided Inquiry Approach. In class, students predicted and discussed the possible products using their knowledge of reaction mechanisms, whereas, in the laboratory, they learned how to safely carry out a reaction with gaseous isobutene and to isolate and identify the two main products. The class-component could be implemented independently as a theoretical exercise -in a "virtual experiment"- simply by presenting the methods and results to students using a guided-inquiry approach. The finding that the simple reaction led to a largely unexpected product, together with open discussions with students covering several theoretical aspects applicable to this reaction, helped to promote critical thinking and provided an effective educational tool to better understand the process of scientific research in chemistry.

\section{TABLE OF CONTENTS/GRAPHIC ABSTRACT}

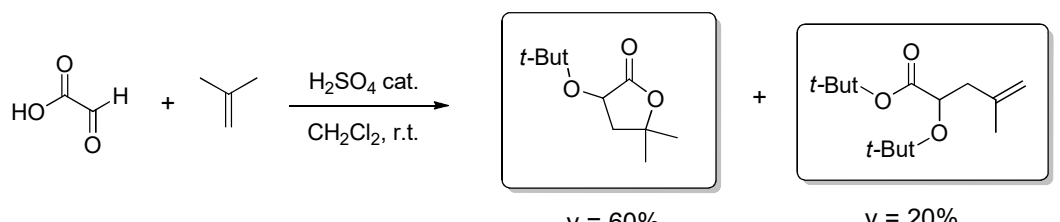

KEYWORDS

Advanced Undergraduates, Organic Chemistry, Laboratory Instruction, Electrophilic Addition, Prins Addition, Elimination Reactions, Synthesis, Guided Inquiry Approach, Glyoxylic Acid, Isobutene, NMR Spectroscopy.

\section{INTRODUCTION AND TIMETABLE}

A research-based experiment was envisioned to promote critical thinking, initiative and curiosity through Guided Inquiry approach. ${ }^{1}$ The laboratory experiment was carried out in groups with 10 volunteers, typically working in pairs, on three independent occasions. The experimental results were also reported by these students to other peers in class, which allowed detailed discussion of theoretical aspects with a further 30 students. 
The students were presented with an easy-to-perform and robust reaction in a two day experiment, for a total laboratory time of 6-7 hrs. This deceptively simple setup was a rich source of theoretical discussion, due to several mechanistic possibilities: the experimental outcome was found by many to be counterintuitive, forcing a reassessment of initial hypotheses. As well as teaching valuable experimental methods and procedures, the overall exercise helped to illustrate clearly the scientific method in organic synthesis.

The Prins reaction is particularly suitable for exploration of mechanisms, because after initial activation of an aldehyde with a Brønsted or Lewis acid, followed by addition of an olefin (Figure 1) to form a cationic intermediate (Figures $2 \& 3$ ), multiple products can be formed. ${ }^{2}$ In this laboratory experiment students had to consider and critically discuss each possible mechanistic pathway.

The exercise was divided into four parts: i) a class discussion to predict the products of the given reaction (1-2 h); ii) an experimental part (6-7 h divided in 2 days, $2-3 \mathrm{~h}$ the first day and 5-6 h the second); iii) structure determination by spectroscopy $(1 \mathrm{~h})$ and iv) presentation of the results to the class and conclusions to determine agreement or disagreement between data and predictions (1-2 h). In the Supporting Information, each part is accompanied with some questions, answers, comments and points of discussions to guide the student's understanding of the exercises.

\section{CLASS DISCUSSION}

The class discussion was ideally suited for peer-discussion in pairs, in which students were first asked to consider the question individually and then to reconsider after a

65 brief discussion with their neighbors. ${ }^{3}$ Initially, students were asked to "list the possible products of a given reaction" (Figure 1), knowing the experimental conditions used.

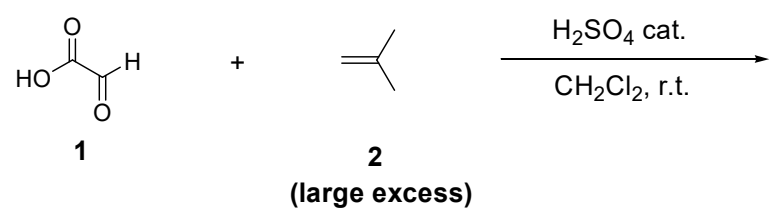

Figure 1. Reaction Scheme.

The reaction between glyoxylic acid (1) and isobutene (2) in dichloromethane catalyzed by sulfuric acid provides an interesting source of discussion, ${ }^{4}$ because glyoxylic acid has two reactive groups, a carboxylic acid and an aldehyde ${ }^{5}$ and two transformations can occur: the alkylation of the acidic moiety through the tertiary carbocation formed

75 after electrophilic addition of isobutene to sulfuric acid, ${ }^{6}$ and the electrophilic addition of the aldehyde to the alkene. ${ }^{2}$ Nearly $90 \%$ of the students involved in this project recognized immediately that isobutene can give electrophilic addition to sulfuric acid to form a tertiary carbocation (Figure 2a), leading to further reaction with the acidic moiety of glyoxylic acid, which ends-up alkylated (Figure S1), whereas only about 60\% realized that isobutene can also act as a nucleophile on the activated aldehyde in a formal Prins addition (Figure $2 \mathrm{~b}$ ). 
a
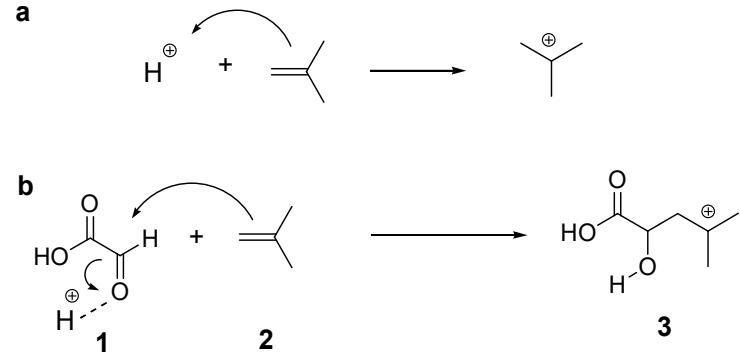

Figure 2. Schematic representation of the Prins addition of isobutene to the aldehyde and of the electrophilic addition of isobutene to sulfuric acid.

The carbocation resulting from the Prins addition can react further in ways that students were asked to critically assess (Figure 3) and to explore experimentally in the laboratory.

Crucial questions used to guide students to consider alternative reactivities and to discuss with them mechanistic aspects are reported in the Notes for Instructors, in the S.I. After class discussion students were able to propose additional reaction pathways and list the possible products (Figure 3), speculating about the more reasonable mechanisms.

3<smiles>CC1CCCCCCCCCCC(C)C1C(=O)O</smiles>

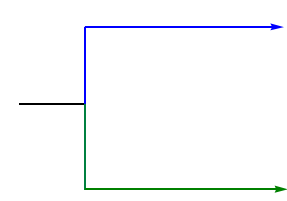

3
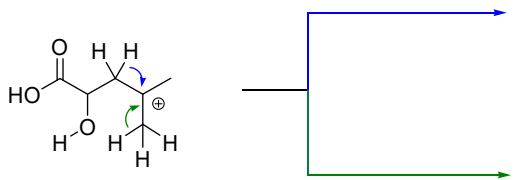<smiles>CC1(C)CC(O)C(=O)O1</smiles><smiles>CC1(C)CC(C(=O)O)O1</smiles><smiles>CC(C)=CC(=O)O</smiles><smiles>C=C(C)CC(O)C(=O)O</smiles>
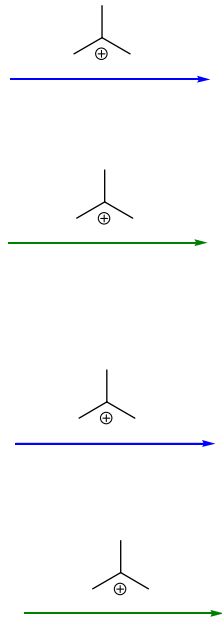<smiles>CC(C)=CC(OC(C)(C)C)C(=O)OC(C)(C)C</smiles>
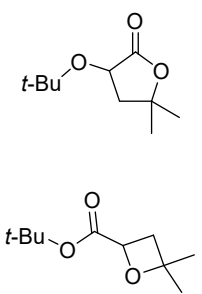

5

6

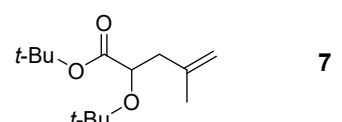

Figure 3. Proposal of reaction pathways and possible products from further reaction of the carbocation intermediate that is formed by formal Prins addition of isobutene to the activated aldehyde.

\section{EXPERIMENTAL PART AND STRUCTURE DETERMINATION}

All the student volunteers were able to set up the experiment correctly using the guidelines provided on all three occasions tested, whereas in the work-up, about 2 in 10 students could not separate efficiently the two main products in their first attempt: a colorless oil and a white powder, whose structures should be determined by NMR. The colorless oil was found to be the olefin $(7)$ (yield $=20 \%$ ), whereas the white powder corresponds to the protected hydroxylactone $(\mathbf{4})$ (yield $=60 \%$ ). The reported yields were obtained from the average yields of the students, which in the best case were $y=70 \%$ 
for (4) and $25 \%$ for (7). The detailed laboratory procedure for students, the notes for instructors, student's results and characterizations are reported in the S.I..

Our experience of student volunteers reporting their results to peers suggests that this experimental part can be provided "virtually", with the methods and results provided by the demonstrator; in this case, the theoretical aspects alone may be sufficient to provide a valuable lesson and shorten the time for the whole exercise to 2-3 hrs, which combines Part 1 (class discussion), Part 3 (structure determination by NMR spectroscopy) and Part 4 (reassessment of initial hypothesis).

\section{HAZARDS}

General laboratory safety procedures, such as wearing safety goggles, a laboratory coat and nitrile gloves, must always be followed. All organic and inorganic chemicals in this experiment are considered hazardous, and direct physical contact must be avoided. Glyoxilic acid is corrosive for the skin and irritating for the eyes. All experiments must be performed in a fume-hood. Particular care should be taken when using methylene chloride as solvent during the reaction and deuterated chloroform as solvent for NMR spectra, as both are suspected carcinogenic compounds. Care must also be taken when using sulfuric acid, a corrosive chemical. Isobutene gas is flammable, and the experiment has to be conducted under a well-ventilated fume hood, in the absence of free flames or heat sources. For a more detailed description of how to use a spray can of isobutene, carefully read the S.I. Cyclohexane and ethyl acetate are both flammable solvents and, additionally, skin contact and inhalation must be avoided. Although dry ice $\left(\mathrm{CO}_{2}\right)$ is not classified as toxic or harmful in accordance with the "Globally Harmonized System of Classification and Labelling of Chemicals (GHS)., prolonged exposure to dry ice can cause severe skin damage through frostbite. Dry ice sublimates into large volumes of carbon dioxide gas which could pose a danger of hypercapnia and should therefore only be exposed to open air in a well-ventilated environment.

The hazard and precautionary statements reported in the S.I. must be carefully read and understood by students, before entering the lab.

\section{RE-ASSESSMENT OF INITIAL HYPOTHESIS}

At the end of the exercise, students involved in the experimental part, were asked to present to the rest of the class their results, formulating conclusions and evaluating their initial hypotheses. Although in the initial class-discussion more than $90 \%$ of the students had correctly expected the formation of the hydroxylactone (4) as major product, upon challenge of which of the two possible minor products (6) or (7) would be expected, about $80 \%$ of students had argued for $(6)$ as the more stable product. However the experimental finding, to the surprise of most students, revealed the less substituted olefin (7) as the minor product. Specific points for discussion are widely reported in the Notes for Instructors in the S.I..

\section{CONCLUSION}

In a typical laboratory situation by practising organic chemists, students were called to predict the possible products of a given reaction with the help of guiding questions. 
The initial class discussion helped the students to define and analize a problem and to improve their critical skills. In the laboratory, students learned how to handle a reaction with gaseous isobutene, to purify products and, finally, to identify these through spectroscopy. At the end of this experiment, the educational achievement for the students was to re-define their hypotheses after the products have been characterized experimentally, in a reiterative process.

Students involved in this experiment found it exciting to work in a typical research situation encountered by chemists that was not a merely a predictable text-book exercise. Judging from student-feedback, this exercise provided the valuable lesson that chemistry remains an empirical science and that even a simple reaction cannot be easily explained or predicted: conclusions and results need to be supported by solid data, which provide a test for hypothesis and provides basis for theoretical models. Conversely, the discussion of ideas and of chemical theory is important in guiding experimental approaches.

\section{ASSOCIATED CONTENT}

Supporting Information. A full experiment section with detailed instructions for students and notes for instructors. List of required reagents with CAS numbers. Full characterization of the purified compounds (4) and (7), with copies of NMR spectra and assignments. This material is available via the Internet at $h t t p: / /$ pubs.acs.org.

\section{AUTHOR INFORMATION}

180 Corresponding Authors

*E-mail: marc.creus@unibas.ch and gaetano.angelici@gmail.com

Notes

The authors declare no competing financial interest.

\section{ACKNOWLEDGMENTS}

The authors would like to thank the financial support from the Swiss National Science Foundation (Grants 200021-116344 and CR22I3_143770), the State Secretariat for Education and Research (Grant C11.0054) and COST Actions TD0905 and CM0902. $\mathrm{MC}$ is particularly grateful for the support from the Holcim Foundation and GA from the Novartis Foundation. The authors would also like to acknowledge Prof. Thomas R.Ward for general encouragement and for allowing the use of laboratory facilities, as well as the students who participated in the practical demonstrations presented here.

\section{REFERENCES}

(1) See for example: Gaddis, B. A.; Schoffstall, A. M. Incorporating Guided-Inquiry Learning into the Organic Chemistry Laboratory. J. Chem. Educ. 2007, 84, 848-851. Mohrig,J. R.; Hammond, C. N.; Colby, D. A. On the successful Use of Inquiry-Driven Experiments into the Organic Chemistry Laboratory. J. Chem. Educ. 2007, 84, 992-998. Mason, D.S. Inquiry Methods in Chemistry. J. Chem. Educ. 2002, 79, 281.

(2) Crane, E. A. ; Scheidt, K. A. Prins-Type Macrocyclizations as an Efficient RingClosing Strategy in Natural Product Synthesis (Review). Angew. Chem. Int. Ed. 2010, 49, 8316 -

200 8326. Dintzner, M. R.; Maresh, J. J.; Kinzie, C. R.; Arena, A. F.; Speltz, T. A Research-Based Undergraduate Organic Laboratory Project: Investigation of a One-Pot, Multicomponent, Environmentally Friendly Prins-Friedel-Crafts-Type Reaction. J. Chem. Educ. 2012, 89, 265-267. 
Delmas, M.; Kalck, P.; Gorrichon, J.-P.; Gaset A. An Easy Student Synthesis of a Substituted 1,3-Dioxane by use of an Ion-exchange Resin as Catalyst. J. Chem. Educ. 2012, 59, 700-701 (3) Smith M.K.; Wood W. B.; Adams W. K.; Wieman C.; Knight J. K.; Guild N.; Su T.T. Why Peer Discussion Improves Student Performance on In-Class Concept Questions. Science 2009, 323. 122-124.

(4) Plusquellec, D.; Le Floc'h, Y.; Paillon-Jegou, D. Réactions de dérivés de l'acide glyoxylique avec des oléfins : synthèses de $\gamma$-lactones $\alpha$-substituée. Bull. Soc. Chim. Fr. 1979, II, 552-558. Jarvis,

210 B. B.; Wells, K. M.; Kaufmann, T. A New Synthetic Route to a-Hydroxy Butyrolactones. Synthesis, 1990, 11, 1079-1082.

(5) Klinger, F. D.; Ebertz, W. Oxocarboxylic Acids. Ullmann's Encyclopedia of Industrial Chemistry, 2012, Wiley-VCH Verlag GmbH \& Co. KGaA, Weinheim. Kerber, R. C.; Fernando, M. S. $\alpha$-Oxocarboxylic Acids. J. Chem. Educ. 2010, 87, 1079-1084

215 (6) Clayden, J.; Greeves, N.; Warren, S.; Wothers, P. Organic Chemistry. 2001, ed.Oxford University Press: Oxford, pp. 503-521. 